\title{
Rapid evolution of phenoloxidase expression, a component of innate immune function, in a natural population of Daphnia magna
}

\author{
Kevin Pauwels, ${ }^{\mathrm{a},{ }^{*}}$ Luc De Meester, ${ }^{\mathrm{a}}$ Stéphanie Put, ${ }^{\mathrm{a}}$ Ellen Decaestecker, ${ }^{\mathrm{a}, \mathrm{b}}$ and Robby Stoks ${ }^{\mathrm{a}}$ \\ ${ }^{a}$ Laboratory of Aquatic Ecology and Evolutionary Biology, Katholieke Universiteit Leuven, Leuven, Belgium \\ b Laboratory of Aquatic Biology, Science \& Technology, Interdisciplinary Research Centre, Katholieke Universiteit Leuven, Kortrijk, \\ Belgium
}

\begin{abstract}
We show rapid microevolution of one component of invertebrate innate immunity, phenoloxidase activity, in a natural population of the water flea Daphnia magna. In Daphnia hatched out of different depths of a sediment core, levels of Daphnia phenoloxidase activity showed genetic variation and increased significantly in less than a decade. We discuss this increase in Daphnia immunity over time in the light of coinciding changes in predator and parasite environment. Together with earlier-found microevolutionary responses in stress protein expression of this Daphnia population, we find simultaneous changes of different defense systems in response towards multiple enemies.
\end{abstract}

There is increasing support for the paradigm that evolution may occur rapidly at an ecological time scale, providing scope for intensive interactions between ecological and evolutionary dynamics (Urban et al. 2008). This has large implications for how we should think about the role of evolution in shaping ecological interactions. Much of current evidence of rapid evolution is based on laboratory studies with one selective factor at a time, which may therefore differ in speed and direction from in situ studies where organisms are exposed to a multitude of selective forces with potentially opposing effects (Reznick and Ghalambor 2005). Yet studies that monitor evolutionary dynamics in situ are scarce (but see Grant and Grant 2002; Reznick et al. 2004). Resurrection ecology, in which evolutionary dynamics are reconstructed by analyzing populations that have been hatched from dormant eggs in layered sediments, is a promising approach to document evolutionary dynamics of natural populations, as it allows digging back in time so that responses to past events can be tracked (Kerfoot and Weider 2004).

Natural enemies may strongly affect fitness and therefore typically impose strong selective forces upon organisms (Crawley 1992), potentially leading to the rapid evolution of defense mechanisms. Arguably, most attention in this regard has gone to predator-induced evolution of defense mechanisms in their prey (Kerfoot and Sih 1987). Much less attention has gone to defense against parasites, which nevertheless represent one of the major threats to any living organism. Even though there is an increasing number of studies that report changes in parasite prevalence and susceptibility through time in natural populations and how this is related to host-parasite coevolutionary dynamics (Decaestecker et al. 2007; Little et al. 2008), these dynamics have not been related directly to evolution in immune defense so far. However, recent studies are providing strong evidence that resistance genes, as a result of the ongoing coevolution with parasites, are some of the

\footnotetext{
*Corresponding author: Kevin.Pauwels@bio.kuleuven.be
}

fastest-evolving genes (Lazzaro 2008; Obbard et al. 2009). In general, research on immune defenses in invertebrates is dominated by mechanistic studies on model organisms, with little attention to natural populations (Little et al. 2005). How the immune system is affected by changing environmental conditions remains a critical research priority (Mydlarz et al. 2006). Rapid evolution of immune defense in natural populations seems likely, as several laboratory studies have been successful in documenting experimental evolution of components of the immune system (Sanders et al. 2005; Schwarzenbach and Ward 2006).

A major component of the invertebrate innate immune system is the prophenoloxidase (proPO) cascade that provides immunity against a large range of pathogens (Soderhall and Cerenius 1998). Upon infection, the inactive proenzyme proPO is converted into phenoloxidase (PO) that catalyzes the production of melanin (Soderhall and Cerenius 1998). The activation of the proPO generates quinonoid intermediates and cytotoxic oxygen radicals that participate in pathogen destruction. As this may cause selfharm, PO is stored in the hemocytes as its inactive proenzyme proPO, which can be activated rapidly upon infection. The proPO cascade has been shown to play a key role in recognition of and defense against microbial infections in invertebrates (Cerenius et al. 2008).

Here we test for microevolution of PO levels in a natural population of the water flea Daphnia magna through time. Natural populations of Daphnia are commonly parasitized by endo- as well as ectoparasites (Stirnadel and Ebert 1997), which are likely strong selective agents because of their negative effect on host reproduction and survival (Gandon et al. 2008; Little et al. 2008). The proPO activating system is an important component of immune function in Daphnia (Mucklow and Ebert 2003). Using resurrection ecology, we here reconstruct levels of PO through time in the Daphnia model system. D. magna clones were hatched from dormant eggs from different depths of a natural population of a pond in Oud-Heverlee 

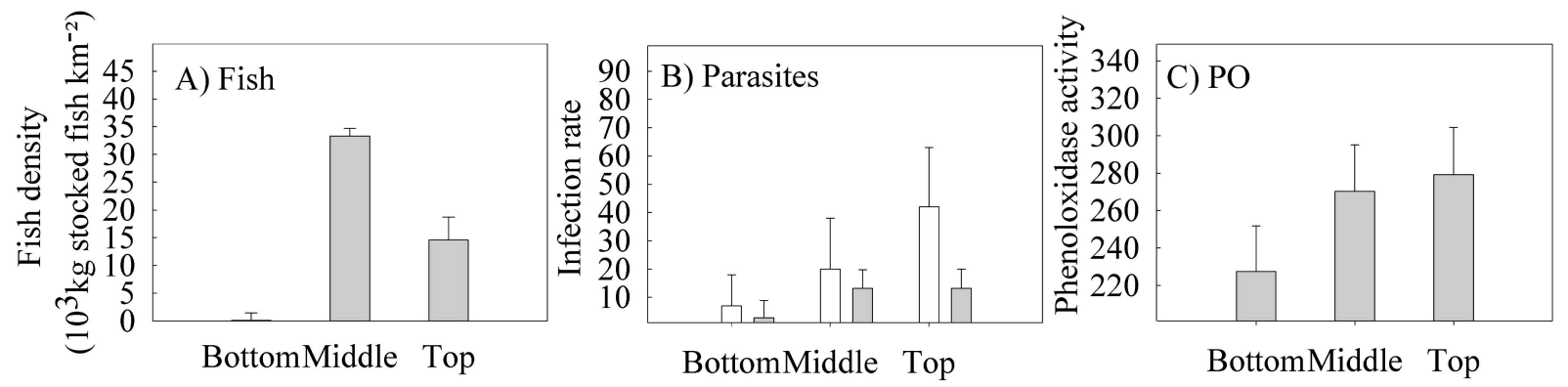

Fig. 1. (A) Levels of stocked fish density, (B) infection rates by parasites after exposure of D. magna to sediment, and (C) phenoloxidase activity levels (after activation of proenzyme prophenoloxidase) of $D$. magna clones for the three periods of the Oud Heverlee pond. Fish stock densities are modified after Cousyn et al. (2001). Infection rates (\%) of epibionts (open bars) are a combination of A. parasiticum and B. rubens and taken from Decaestecker et al. (2004); infection rates of gut parasites (shaded bars) are a combination G. intestinalis and $O$. colligata (E. Decaestecker unpubl. data). Means are given with 1 SE.

(Belgium) where strong temporal changes in fish predation pressure (Cousyn et al. 2001) as well as an increase in parasitic load (Decaestecker et al. 2004; E. Decaestecker unpubl. data) have been documented (Fig. 1A,B). Both selection pressures have had a major effect on the evolutionary trajectory of the population, causing microevolutionary changes in phototactic behavior (Cousyn et al. 2001) and levels of heat shock protein expression (Pauwels et al. 2007).

\section{Methods}

Study system - We used D. magna clones hatched from a dormant egg bank of a former fish culture pond in OudHeverlee (Belgium). Dormant eggs were isolated from each of the three main periods in fish-stocking history in this pond: bottom (ca. 1970-1972) with low predation (newly created pond, prior to fish stocking), middle (ca. 19761979) with high predation (stocking of up to $30,000 \mathrm{~kg} \mathrm{~km}^{-2}$ of planktivorous and benthivorous fish), and top (19881990 ) with reduced predation (reduced levels of stocking; Cousyn et al. 2001; Fig. 1A). After hatching, 12 clones of each period were kept under standardized conditions in the laboratory for several generations prior to the experiments, so there is no interference from prior experience or exposure to predators and parasites in their natural habitat. Moreover, to obtain independent replicates per clone, we set up five clonal lines per clone, which were raised separately in optimal conditions for two generations. Then, for each clonal line, three individuals, all born within a 24-h interval, were raised till second-instar adults for assessment of (pro)PO levels.

Analysis of (pro)PO levels-Levels of (pro)PO in the Daphnia hemolymph were measured photometrically as the production rate of dopachrome after oxidation of L-3,4dihydroxyphenylalanine (L-dopa) following the protocol by Mucklow and Ebert (2003). Hemolymph was collected in a microcapillary tube after pricking the Daphnia with a needle close to the heart. Hemolymph of three animals was pooled in order to obtain a final volume of $2 \mu \mathrm{L}$, which was transferred in $150 \mu \mathrm{L}$ of phosphate-buffered saline (PBS) buffer $\left(0.15 \mathrm{~mol} \mathrm{~L}^{-1} \mathrm{NaCl}, 10 \mathrm{mmol} \mathrm{L}-1 \mathrm{Na}_{2} \mathrm{HPO}_{4} \cdot 2 \mathrm{H}_{2} \mathrm{O}\right.$, pH 7.4 with o-phosphoric acid). Then $50 \mu \mathrm{L}$ of sample was added to $225 \mu \mathrm{L}$ of $20 \mathrm{mmol} \mathrm{L}^{-1} \mathrm{~L}$-dopa and the absorbance of dopachrome was measured at $475 \mathrm{~nm}$. Samples were run in duplicate and mean values were taken for further analysis. $\mathrm{PO}$ activity of the samples was calculated as the increase of the absorbance values after $4.5 \mathrm{~h}$ corrected for changes in the control (PBS and L-dopa, without hemolymph; Mucklow and Ebert 2003). The total PO was assayed after the activation of proPO with $5 \mu \mathrm{L}$ of chymotrypsin to active PO. As both measures showed parallel responses, we only present the data on total PO, as for studying evolutionary patterns in constitutive immune response, including proPO is the most relevant (Jacot et al. 2005). We will further only use the term PO levels to refer to total PO.

Parasite induction experiments-To test the idea that PO can be directly up-regulated by the presence of parasites, we performed two experiments with four randomly selected clones of the study population. In a first experiment, we correlate infection rates with PO levels in host genotypes when exposed to a mixed sediment sample of the OudHeverlee pond (taken from a depth roughly corresponding to the period 1970-1990). Daphnia exposed to sediment samples that contain viable parasite spores will become infected (Decaestecker et al. 2004). Five juveniles were placed in $50-\mathrm{mL}$ plastic vials, containing $20 \mathrm{~mL}$ of sediment and $20 \mathrm{~mL}$ of dechlorinated tap water, within $12 \mathrm{~h}$ after birth. For each clone, 20 replicate groups of five individuals were kept. After $6 \mathrm{~d}$, animals were transferred to fresh vials containing dechlorinated tap water without sediment. From then onwards, half of the medium was refreshed every day, and newborns were removed. After $21 \mathrm{~d}$, parasite infections were determined and hemolymph was taken for PO measurement. Infection rates were scored under an optical stereomicroscope (400X magnification) for the most abundant resident parasites (Decaestecker et al. 2004; E. Decaestecker unpubl. data): the epibionts Amoebidium parasiticum and Brachionus rubens, and, after dissection of gut tissue, the gut parasites Glugoides intestinalis and Ordospora colligata, which were for convenience pooled in further analyses.

In a second experiment we compared PO levels between exposed and nonexposed individuals of the same host clones. For this experiment, we chose the epibiont $B$. rubens, as this was the parasite for which we detected the 


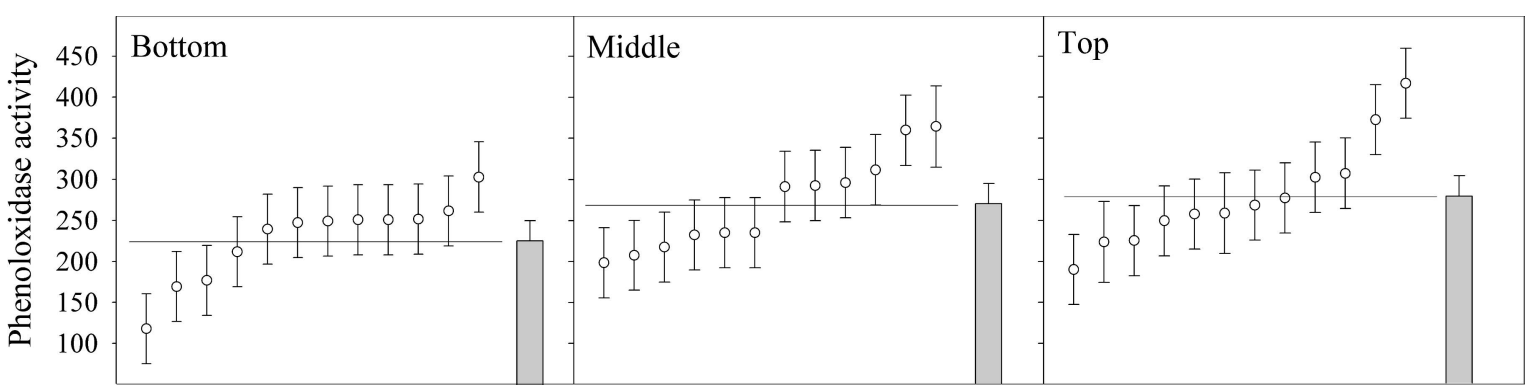

Fig. 2. Phenoloxidase levels for 12 D. magna clones from each of three periods differing in fish-stocking history (bottom [19701972]; middle [1976-1979]; top [1988-1990]). Within each subpopulation, the clones are ordered according to increasing levels of PO. Bars indicate subpopulation means (based on clonal means $\pm 1 \mathrm{SE}$ ). Shown are total PO levels obtained after activation of the proenzyme prophenoloxidase.

highest infection rate and a positive correlation with PO levels in the first experiment (see results). Daphnia were exposed to the epibiont $B$. rubens through horizontal transmission from infected Daphnia and compared with a control treatment. Five newborn juveniles (within $12 \mathrm{~h}$ after birth) were placed in $100-\mathrm{mL}$ jars containing five heavily infected animals ( $>$ five adult epibionts per Daphnia), separated from the experimental animals by a mesh (size $120 \mu \mathrm{m})$. This mesh did not obstruct free movement of epibionts. After $6 \mathrm{~d}$ animals were transferred to fresh jars. From then onwards, half of the medium was refreshed every day, and newborns were removed. After $21 \mathrm{~d} B$. rubens infections were determined and hemolymph was taken for PO measurement.

Statistical analyses-Constitutive levels of PO were analyzed using analysis of variance (ANOVA) with period (fixed) and clone (random) nested in period as independent variables. Evolutionary rates were expressed as Haldanes (Kinnison and Hendry 2001) and based on the assumption that 1 calendar year is equal to one generation (midpoints of periods are used to calculate number of generations), which is a reasonable assumption given the cyclic parthenogenetic reproduction cycle of $D$. magna involving a yearly bout of sexual reproduction. Broad-sense heritabilities are estimated by clonal repeatability analysis (Lynch and Walsh 1998).

Effects of infection rates on PO levels were analyzed using separate analyses of covariance per parasite with clone as independent variable, infection rate as covariate and PO level as dependent variable. Infection rates were arcsin square root transformed. Interactions with the covariate were never significant (all $p>0.50$ ) and were dropped from the final models. The effect of $B$. rubens infection on PO levels was tested using a two-way ANOVA with parasite exposure treatment and clone as independent variables and PO level as dependent variable. All analyses were performed using Statistica 9.0.

\section{Results}

Levels of PO changed through time (period: $F_{2,105}=$ $4.95, p=0.009$ ), with lower levels in the bottom compared to the middle and top periods (Duncan post hoc, $p<0.023$; $p<0.004)$, which did not differ from each other $(p=0.47$;
Figs. 1C, 2). The associated rates of evolutionary change in PO levels were 0.11 Haldanes between bottom and middle and 0.01 Haldanes between middle and top period. There was genetic variation for PO levels within the subpopulations of each period (clone[period]: $F_{33,105}=1.75, p=$ $0.018)$. The associated overall heritability $\left(\mathrm{H}^{2}\right)$ was 0.16 , showing a trend from more clonal variation in the bottom $(0.17)$ to less variation in the top subpopulation $(0.05)$.

After $21 \mathrm{~d}$ in the first parasite induction experiment, animals were infected by $A$. parasiticum $(96.2 \%), B$. rubens $(67.1 \%)$, and gut parasites $(10.2 \%)$. No evidence of clonal differences in infection rate was found for any of the parasites, although there was a tendency for clonal differences in $A$. parasiticum (A. parasiticum, $F_{3,75}=2.47$, $p=0.07 ; B$. rubens, $F_{3,75}=1.30, p=0.28$; gut parasites, $F_{3,75}=0.44, p=0.73$ ). Infection rates with $B$. rubens (slope $\left.=197, \mathrm{SE}=52 ; F_{1,74}=14.15, p=0.0003\right)$ as well as with gut parasites $\left(\right.$ slope $=372, \mathrm{SE}=78 ; F_{1,74}=22.84, p<$ 0.0001 ) showed a positive covariation with the levels of PO, but no correlation was found for $A$. parasiticum (slope = $\left.-62, \mathrm{SE}=56 ; F_{1,74}=1.23, p=0.27\right)$. After $21 \mathrm{~d}$ in the second parasite induction experiment, Daphnia infected with B. rubens (PO activity $\pm 1 \mathrm{SE}=510 \pm 42$ activity units) showed higher PO levels compared to control animals (PO activity $\pm 1 \mathrm{SE}=338 \pm 41$ activity units) that were not infected $\left(F_{1,21}=8.55, p=0.008\right)$. Clones did not differ in PO $\left(F_{3,21}=0.83, p=0.49\right)$ or in the response to the epibiont infection $\left(F_{3,21}=0.97, p=0.43\right)$.

\section{Discussion}

Levels of PO increased through time between the different subpopulations, each corresponding to a different time period of the same natural water flea population. Neutral microsatellite markers on this population indicated that the subpopulations separated in time belong genetically to one continuous population (Cousyn et al. 2001). Further, based on the high number of dormant eggs, even in the subpopulation with high fish predation pressure the estimated number of genotypes is $>400 \times 10^{6}$ (Cousyn et al. 2001), and the buffering capacity of the dormant egg bank, it seems unlikely that genetic drift could have caused this pattern. Genetic differentiation in PO levels therefore likely reflects an in situ microevolutionary response due to changing local selection pressures (see also Cousyn et al. 
2001 for antipredator behavior). The high rate (Kinnison and Hendry 2001) of microevolutionary change in PO levels between the bottom and the middle period combined with overall rather low heritabilities suggests strong selection pressures were at work.

As PO is a major component of the invertebrate immune system (Söderhall and Cerenius 1998) and found to be upregulated in parasitized Daphnia (this study), it is tempting to relate the temporal dynamics in PO levels to the temporal dynamics in parasite pressure. We consider this temporal dynamics in parasite pressure as real and not an artifact of ageing parasite spores. Daphnia exposed to sediment containing parasite spores from different periods reveal an increased parasite load between the succeeding time periods, mainly by the epibiont $A$. parasiticum (Decaestecker et al. 2004) and gut parasites (E. Decaestecker unpubl. data; Fig. 1B). One may argue that this pattern can be caused by a decreased viability of parasite spores with age rather than reflecting changes in parasite load through time. An analysis of several sediment cores revealed, however, widely different patterns of change in spore loads in different habitats, including patterns that cannot be explained by a loss of infectivity with age (Decaestecker et al. 2004, 2007). In addition, the results of Decaestecker et al. (2007) show no decline in the overall capacity to infect hosts with age of the parasite spores. There are thus no indications that accumulated heritable damage in the parasite spores during dormancy results in deteriorated fitness or infection capacities within the time span of the investigated sediment core. The increased parasite load with time observed in the present study thus can be interpreted to reflect changes in parasite pressure through time.

In line with a suggested role of parasite pressure in shaping PO dynamics, both variables increase between the first and second time period. Further, both parasite induction experiments indicate an up-regulation upon exposure to (ecto) parasites, supporting the involvement of the proPO cascade in the defense against parasites in the studied host species. B. rubens is a relatively large rotifer that attaches to the host carapax, and in this way may be likely to cause small injuries. Induction of PO has been shown after wounding in Daphnia (Mucklow and Ebert 2003). Gut parasites are intracellular parasites of the gut epithelium and have been shown to induce PO in bumblebees (Brown et al. 2003). Despite these indications for a direct role of parasites in shaping PO dynamics, we acknowledge that the resurrection ecology approach, albeit very powerful in identifying evolutionary responses (Jeppesen et al. 2001), remains correlative in nature when explaining these responses. Therefore, the presented causal connection between the evolution of PO levels and the parallel change in parasite pressure remains to some extent speculative, yet backed up by both parasite induction experiments and more likely than some other alternative hypotheses, as we will discuss further.

The combination of predation risk and parasites together can have a strong effect on population dynamics (Hatcher et al. 2006), and if only parasites would play a role in the evolution of PO levels, we would expect a continuous increase from the bottom to the top period. We therefore hypothesize that the temporal pattern for PO may have been modulated by the associated changes in fish predation pressure. In response to predation risk by fish, Daphnia in our study population exhibit an induced negative phototactic behavior (Cousyn et al. 2001), meaning they reside closer to the sediment, which contains parasite spore banks (Decaestecker et al. 2004). This increases infection rates and infection intensities with parasites (Decaestecker et al. 2002). Therefore, the fish-induced diel vertical migration behavior may have made the selection imposed by the parasites much stronger. Between the middle and the top period, the fish predation pressure reduced again and the Daphnia showed a less strong diel vertical migration response (Cousyn et al. 2001), which may explain why PO levels did not continue to increase. An alternative explanation is offered by the difference in the abundance of epibionts and gut parasites through time. Although the abundance of epibionts kept increasing from the bottom to the top, this was not the case for gut parasites. Both studied gut parasites are able to alter the outcome of competition among host genotypes and are expected to act as a strong selective force (Capaul and Ebert 2003). In contrast, epibionts show low host specificity and generally have less effect on host fecundity, although they may reduce host mobility and interfere with feeding and predator escape behavior (Chiavelli et al. 1993). Epibionts alone therefore might be a weaker stressor than parasites, and less effective in increasing constitutive levels of immune defense. This may also explain that only the larger epibiont, Brachionus, induced an increase of PO upon infection.

Note that so far we considered temporal changes in predation pressure only to play an indirect role, by intensifying parasite pressure, and no direct role in driving the temporal PO dynamics. This is motivated by the fact that although fish predation also increased from the first to the second period, previous work showed no direct upregulation of PO under predation risk in Daphnia (K. Pauwels unpubl. data). During that time frame the resident Daphnia population was faced with a drastic shift from no risk of fish predation towards a severe fish predation pressure (Cousyn et al. 2001). This resulted in a shift towards genotypes that showed better protection against predation, with major changes in migration behavior (Cousyn et al. 2001) and life history variables (B. Jansen unpubl. data). One may argue that PO levels changed in association with traits that allowed predation avoidance. PO is shown to be costly, and life history shifts can influence the PO levels through changes in energy allocation (Pauwels et al. 2010). Yet Daphnia like other prey organisms is, if anything, considered to contain less energy under predation risk, making an up-regulation as a result of energetic reallocation under increasing predation risk through time unlikely. Further, PO levels do not decrease again towards the top period, indicating that any possible correlation with predation risk cannot explain the whole pattern.

In several studies of invertebrates, PO activity has been shown to be a good measure of defense against parasites and pathogens, suggesting it to be a good indicator of immuno- 
competence (Butt and Raftos 2008). Equally, in Daphnia it was found that clonal variation in PO activity covaried with differences in resistance against parasites (Mucklow and Ebert 2003). Yet this covariation pattern disappeared when the source populations of the different clones were taken into account (Mucklow et al. 2004). This was interpreted as PO being only a part of the multivariate immune response, which on its own cannot be used for predicting general immunocompetence in Daphnia. Future studies should therefore include more components of innate immune function and consider potential trade-offs between them.

Summarizing, we documented rapid microevolution of a component of innate immunity, more in particular PO activity, in a natural population of the water flea $D$. magna. Our study thereby extends previous work showing experimental evolution of immune components (Sanders et al. 2005; Schwarzenbach and Ward 2006) toward realistic field conditions. The study population showed adaptive microevolutionary responses in behavior to changes in predation risk (Cousyn et al. 2001), while at the same time also showing microevolutionary responses in stress protein (Pauwels et al. 2007) and PO levels (present study) that parallel changes in parasite load. These combined results illustrate that natural Daphnia populations have the ability to genetically adapt at the same time to changes in several important enemies, and do so simultaneously with different defense systems.

\section{Acknowledgments}

We thank two anonymous referees for their comments on an earlier version of the manuscript. K.P. was supported by a scholarship from the Institute for the Promotion of Innovation through Science and Technology in Flanders (IWT-Vlaanderen). E.D. is a part-time postdoctoral fellow of the Fund for Scientific Research of the K. U. Leuven. Financial support came from research grants (G.0269.04) from Research Foundation-Flanders and projects $(\mathrm{OT} / 04 / 23)$ and $(\mathrm{GOA} / 2008 / 06)$ from the $\mathrm{K}$. U. Leuven Research Fund.

\section{References}

Brown, M. J. F., Y. Moret, and P. Schmid-Hempel. 2003. Activation of host constitutive immune defence by an intestinal trypanosome parasite of bumble bees. Parasitology 126: $253-260$, doi: $10.1017 / \mathrm{S} 0031182002002755$

Butt, D., And D. Raftos. 2008. Phenoloxidase-associated cellular defence in the Sydney rock oyster, Saccostrea glomerata, provides resistance against QX disease infections. Dev. Comp. Immunol. 32: 299-306, doi:10.1016/j.dci.2007.06.006

Capaul, M., and D. Ebert. 2003. Parasite-mediated selection in experimental Daphnia magna populations. Evolution 57: 249-260.

Cerenius, L., B. L. Lee, and K. Soderhall. 2008. The proPOsystem: Pros and cons for its role in invertebrate immunity. Trends Immunol. 29: 263-271, doi:10.1016/j.it.2008.02.009

Chiavelli, D. A., E. L. Mills, and S. T. Threlkeld. 1993. Host preference, seasonality, and community interactions of zooplankton epibionts. Limnol. Oceanogr. 38: 574-583.

Cousyn, C., L. De Meester, J. K. Colbourne, L. Brendonck, D. Verschuren, and F. Volckaert. 2001. Rapid, local adaptation of zooplankton behavior to changes in predation pressure in the absence of neutral genetic changes. Proc. Natl. Acad. Sci. USA 98: 6256-6260, doi:10.1073/ pnas. 111606798
Crawley, M. 1992. Natural enemies - the population biology of predators, parasites and diseases, 1st ed. Blackwell.

Decaestecker, E., L. De Meester, and D. Ebert. 2002. In deep trouble: Habitat selection constrained by multiple enemies in zooplankton. Proc. Natl. Acad. Sci. USA 99: 5481-5485, doi:10.1073/pnas.082543099

, S. Gaba, J. A. M. Raeymaekers, R. Stoks, L. Van Kerckhoven, D. Ebert, and L. De Meester. 2007. Hostparasite "Red Queen" dynamics archived in pond sediment. Nature 450: 870-873, doi:10.1038/nature06291

C. Lefever, L. De Meester, and D. Ebert. 2004. Haunted by the past: Evidence for dormant stage banks of microparasites and epibionts of Daphnia. Limnol. Oceanogr. 49: $1355-1364$.

Gandon, S., A. Buckling, E. Decaestecker, and T. Day. 2008. Host-parasite coevolution and patterns of adaptation across time and space. J. Evol. Biol. 21: 1861-1866, doi:10.1111/ j.1420-9101.2008.01598.x

Grant, P. R., AND B. R. Grant. 2002. Unpredictable evolution in a 30-year study of Darwin's finches. Science 296: 707-711, doi:10.1126/science.1070315

Hatcher, M. J., J. T. A. Dick, And A. M. Dunn. 2006. How parasites affect interactions between competitors and predators. Ecol. Lett. 9: 1253-1271, doi:10.1111/j.1461-0248.2006.00964.x

Jacot, A., H. Scheuber, J. Kurtz, AND M. W. G. BrinKhof. 2005. Juvenile immune system activation induces a costly upregulation of adult immunity in field crickets Gryllus campestris. Proc. R. Soc. B Biol. Sci. 272: 63-69, doi:10.1098/rspb.2004.2919

Jeppesen, E., P. Leavitt, L. De Meester, and J. P. Jensen. 2001. Functional ecology and palaeolimnology: Using cladoceran remains to reconstruct anthropogenic impact. Trends Ecol. Evol. 16: 191-198, doi:10.1016/S0169-5347(01)02100-0

Kerfoot, W. C., AND A. Sih. 1987. Predation: Direct and indirect impacts on aquatic communities. Univ. Press of New England.

- AND L. J. WeIDER. 2004. Experimental paleoecology (resurrection ecology): Chasing Van Valen's Red Queen hypothesis. Limnol. Oceanogr. 49: 1300-1316.

Kinnison, M. T., AND A. P. Hendry. 2001. The pace of modern life II: From rates of contemporary microevolution to pattern and process. Genetica 112: 145-164, doi:10.1023/ A: 1013375419520

Lazzaro, B. P. 2008. Natural selection on the Drosophila antimicrobial immune system. Curr. Opin. Microbiol. 11: 284-289, doi:10.1016/j.mib.2008.05.001

Little, T. J., W. Chadwick, and K. Watt. 2008. Parasite variation and the evolution of virulence in a Daphniamicroparasite system. Parasitology 135: 303-308, doi:10.1017/S0031182007003939

, D. Hultmark, And A. F. Read. 2005. Invertebrate immunity and the limits of mechanistic immunology. Nat. Immunol. 6: 651-654, doi:10.1038/ni1219

LyNCH, M., AND B. WALSH. 1998. Genetics and analysis of quantitative traits, 1st ed. Sinauer Associates, Inc.

Mucklow, P. T., AND D. Ebert. 2003. Physiology of immunity in the water flea Daphnia magna: Environmental and genetic aspects of phenoloxidase activity. Physiol. Biochem. Zool. 76: 836-842, doi: $10.1086 / 378917$

, D. B. Vizoso, K. H. Jensen, D. Refardt, and D. Ebert. 2004. Variation in phenoloxidase activity and its relation to parasite resistance within and between populations of Daphnia magna. Proc. R. Soc. B Biol. Sci. 271: 1175-1183, doi:10.1098/rspb.2004.2707

Mydlarz, L. D., L. E. Jones, and C. D. Harvell. 2006. Innate immunity environmental drivers and disease ecology of marine and freshwater invertebrates. Annu. Rev. Ecol. Evol. Syst. 37: 251-288, doi:10.1146/annurev.ecolsys.37.091305.110103 
Obbard, D. J., J. J. Welch, K. W. Kim, and F. M. JigGins. 2009. Quantifying adaptive evolution in the Drosophila immune system. PLoS Genet. 5: e1000698, doi:10.1371/journal.pgen. 1000698

Paumels, K., R. Stoks, and L. De Meester. 2010. Enhanced antipredator defence in the presence of food stress in the water flea Daphnia magna. Funct. Ecol. 24: 322-329, doi:10.1111/ j.1365-2435.2009.01641.x

, E. Decaestecker, and L. De Meester. 2007. Evolution of heat shock protein expression in a natural population of Daphnia magna. Am. Nat. 170: 800-805, doi:10.1086/521956

Reznick, D. N., M. J. Bryant, D. Roff, C. K. Ghalambor, and D. E. Ghalambor. 2004. Effect of extrinsic mortality on the evolution of senescence in guppies. Nature 431: 1095-1099, doi:10.1038/nature02936

, ANd C. K. Ghalambor. 2005. Selection in nature: Experimental manipulations of natural populations. Integr. Comp. Biol. 45: 456-462, doi:10.1093/icb/45.3.456

Sanders, A. E., C. Scarborough, S. J. Layen, A. R. KraAijeveld, AND H. C. J. Godfray. 2005. Evolutionary change in parasitoid resistance under crowded conditions in Drosophila melanogaster. Evolution 59: 1292-1299.
Schwarzenbach, G. A., And P. I. Ward. 2006. Responses to selection on phenoloxidase activity in yellow dung flies. Evolution 60: 1612-1621.

Söderhall, K., AND L. Cerenius. 1998. Role of the prophenoloxidase-activating system in invertebrate immunity. Curr. Opin. Immunol. 10: 23-28, doi:10.1016/S0952-7915(98)80026-5

Stirnadel, H. A., And D. Ebert. 1997. Prevalence, host specificity and impact on host fecundity of microparasites and epibionts in three sympatric Daphnia species. J. Anim. Ecol. 66: 212-222, doi: $10.2307 / 6023$

Urban, M. C., AND others. 2008. The evolutionary ecology of metacommunities. Trends Ecol. Evol. 23: 311-317, doi:10.1016/j.tree.2008.02.007

Associate editor: Michael R. Landry

Received: 08 September 2009

Accepted: 15 February 2010 Amended: 01 March 2010 